\title{
Respiratory, Hepatic, Renal and Hematological Disorders Among Adolescent Females Environmentally Exposed To Pesticides, Menoufia Governorate, Egypt
}

\author{
Eman Abdel-Azeem Salem \\ Menoufia Faculty of Medicine \\ Olfat Mohamed Hendy \\ Menoufia University Liver Institute \\ Diane S Rohlman \\ The University of lowa \\ Asmaa Abdel-Rehim Abdel-Latif \\ Menoufia University Faculty of Medicine \\ Aziza Saad Elbadry \\ Menoufia University Faculty of Medicine
}

Gaafar Mohamed Abdel-Rasoul ( $\sim$ gaafar237@yahoo.com )

Menoufia University Faculty of Medicine https://orcid.org/0000-0001-6007-9521

\section{Research Article}

Keywords: Environmental Pesticides' Exposure, Adolescent Females, Respiratory, Hepatic, Renal and Hematological

Disorders, AChE

Posted Date: November 10th, 2021

DOI: https://doi.org/10.21203/rs.3.rs-1023694/v1

License: (c) (i) This work is licensed under a Creative Commons Attribution 4.0 International License. Read Full License

Version of Record: A version of this preprint was published at Environmental Science and Pollution Research on January 24th, 2022. See the published version at https://doi.org/10.1007/s11356-021-18420-3. 


\section{Abstract}

Adolescent females are often environmentally exposed to pesticides by living near agricultural fields, by using pesticides at home, or by having contact with contaminated clothes and pesticide application work tools. This study assessed respiratory, hepatic, renal and hematological health disorders that might arise due to environmental exposure to pesticides among adolescent females. A cross-sectional study was conducted with 100 adolescent females environmentally exposed to pesticides who had one or more of their family members working as seasonal pesticides' applicators and 50 non- exposed (control) adolescent females from Menoufia governorate, Egypt. The studied period of pesticide application season of cotton crop was from May $1^{\text {st }}$ to the end of September 2017. Participants completed a self-administered questionnaire about pesticide exposure and respiratory, hepatic, renal and hematological disorders. In addition, serum acetyl cholinesterase (AChE), spirometry, complete blood count, liver and kidney functions' tests were measured pre and post pesticide application season. The control adolescent females had a higher AChE activity, a lower prevalence of respiratory symptoms and higher means of spirometric measurements than the exposed group. During the pre and postseason, the exposed group presented a prevalence of $(6 \%, 24 \%)$ for cough, $(4 \%, 11 \%)$ for rhinitis, and $(6 \%, 26 \%)$ for dyspnea during the pre and postseason; respectively. In addition, there was a decrease in means of spirometric measurements $\left(\mathrm{FEV}_{1} \%, \mathrm{FEV}_{1} / \mathrm{FVC}\right.$, FEF 25 -

$75 \%$ and $\mathrm{PEF} \%$ ) in post season compared to preseason among the exposed group. Also, there were significant associations between (AChE) activity and both the prevalence of respiratory manifestations and spirometric measurements among the exposed females. On the other hand, there were significantly increased red blood corpuscles (RBCs) and lymphocytes counts, and a significantly lower mean hemoglobin level among the exposed group (post season) than each of their pre-season values and the control group $(P<0.05)$. AChE level, total protein, albumin and albumin/globulin (A/G) ratio levels were significantly lower, while SGPT, SGOT, and globulin, blood urea and serum creatinine mean levels were significantly higher among the exposed group (post season) than either of their pre-season or the control group $(P<0.05)$. There was a positive correlation between AChE level and all studied $C B C$ parameters for the exposed group reaching a significant level with basophils $(P<0.05)$. Also, there was a negative correlation between AChE level and each of SGPT, SGOT, ALP, globulin, blood urea and serum creatinine for the exposed group reaching a significant level with each of SGPT and SGOT $(P<0.05)$. At the same time, a nonsignificant positive correlation was found between $A C h E$ level and each of total protein, albumin and $A / G$ ratio $(P>0.05)$. So, environmental exposure to organophosphorus pesticides has a detrimental impact on respiratory, hepatic, renal and hematological systems of adolescent females living in rural districts at Menoufia governorate. Educational and training intervention programs on pesticide handling and safety precautions are recommended for protecting both pesticides' workers and their family members who might be exposed.

\section{Introduction:}

Pesticides are toxic chemicals that are widely used throughout the world in agriculture on crops as well as for domestic purposes for mosquito and cockroach control operations (Rani et al.; 2017). They are semi-volatile, even nonvolatile substances, and surface deposition occurs rapidly after use. These substances enter the body through the skin, the digestive and the respiratory tracts (Baldi et al., 2012).

Environmental pollution results from pesticide use as more than $98 \%$ of applicated insecticides and $95 \%$ of herbicides affect other destinations than their required target species (Miller, 2004).

Children and adolescents constitute a high risk vulnerable group on exposure to pesticides (than adults) due to the expected occurrence of significant anatomical, maturational and physiological changes as they are in the 
developmental stage of body systems (Abdel-Rasoul et al., 2008). This is evidenced by the health effects of pesticides in adults which come from studies of occupationally exposed adult males. Scanty information is known concerning pesticide-related health effects in adolescent females, and there may be reproductive toxicity due to sexspecific risk differences (Rohlman et al., 2007).

The responsibility of environmental factors explained the relationship between the presence of allergic asthma and pesticides' exposure. A lower potentiated vagally induced bronchoconstriction threshold level and increased formation of proinflammatory cytokines in allergen sensitized guinea pigs were initiated by organophosphorus insecticides (Proskocil et al. 2010).

A higher prevalence of upper respiratory tract infections and 'influenza-like' symptoms was observed in workers occupationally exposed to organophosphates (OPs) and was associated with a decrease in serum and red blood corpuscle cholinesterase activity (Kamanyire and Karalliedde 2004). Also, pesticide production workers and farmers exposed to organophosphorus (OP) and carbamates (CM) are reported to have significantly decreased pulmonary functions, increased wheezing, and increased risk for asthma compared to controls. Moreover, workers employed in the manufacture of chlorfenvinphos showed decreased lung functions and weakened respiratory muscles than controls (Mamane et al. 2015).

The liver represents a major center in the detoxification process as it faces the challenge of maximum exposure to xenobiotics and their metabolic by-products. The overall balance between the degree of oxidative stress and the antioxidant capability determines the susceptibility of liver tissues to this stress resulting from exposure to pesticides (Khan et al., 2005)..

Acute renal failure and multiple organ dysfunction syndromes, suggesting that high intratubular organophosphorus pesticides (OP) concentrations and hypovolemia might be responsible for acute kidney injury (AKI) following exposure to organophosphorus pesticides (OP), which is currently defined by either change in serum creatinine or urine output. Persistent decline of renal function without recovery can lead to end-stage renal disease and increase the short-term and long term risk of death (Lee et al., 2015).

Many steps in heme biosynthesis are inhibited by pesticide residues. Another possible mechanism is binding of organophosphate pesticides on iron, followed by a lack of incorporation of iron in hemoglobin (Abu Mourad, 2005). This effect may increase to reach to aplastic anemia (Adad et al., 2015).

According to the Egyptian culture no females are allowed to work as pesticide applicators, hence their exposure to pesticides could be exclusively environmental either by living nearby agriculture fields, domestic use of home pesticides, or to handling clothes of their family members (brothers or fathers) that are contaminated with pesticides' residues.

To our knowledge, no studies have been carried out examining the effects of exposure to pesticides among environmentally exposed adolescent females in Egypt. The aim of this study was to assess respiratory, hepatic, renal and hematologic health disorders among studied adolescent females that might arise due to environmental exposure to pesticides.

\section{Subjects And Methods:}

\section{Study Design:}


This was a cross-sectional study.

\section{Place and Duration of the Study:}

The study was conducted at three randomly chosen districts (Shebin El-Kom, El-Bagour and Menouf) out of the ten districts in Menoufia governorate, Egypt. The studied period of pesticide application season of cotton crop was from May $1^{\text {st }}$ to the end of September 2017.

\section{Study Sample:}

The exposed group consisted of 100 adolescent females (aged from 9-18 years) who had one or more of their family members working as seasonal pesticide applicators in cotton fields and / or working privately with their own backpack sprayers applying pesticides to other crops throughout the year. The pesticide exposed female adolescents' homes were located less than 1000 meters from the agricultural fields

The control group included 50 adolescent females that were matched with the exposed group regarding age, residence, socioeconomic standard, educational level and their family members were never involved in pesticides' application. Their homes were located more than 1000 meters away from the agricultural fields.

All participants in both groups were never been married. Chosen adolescent females having a history of chronic medical disorders (i.e., diabetes, hypertension, chest, liver, kidney or blood disease) were excluded from the study.

\section{Study Methods:}

Each participant completed the following at two time points, prior to the application season (pre-season) and after the application season ended (post-season):

\section{I: A self-administered predesigned questionnaire which included.:}

a. Personal data as age, residence, level of education, the distance of house from fields and past medical history of diseases.

b. Pesticide exposure history as applying pesticides at home, use of empty pesticide containers, handling contaminated clothes of their relatives, existing in fields within 3 days after pesticide spraying.

c. Occupational history of their pesticide applicators' relatives: working days and duration of work.

d. Respiratory symptoms as rhinitis, cough, expectoration dyspnea, wheezes and chest pain.

\section{II: Spirometric measurements:}

Spirometric tests were done using the MEE Spiro PFT touch, Germany. Participants performed up to three maneuvers according to the American Thoracic Society guidelines for pulmonary function tests. Percent predicted values were calculated as a percent of the participant measured value to a predicted value of that participant. The predicted values were those of Knudson's method (Quadrelli et al. 1999). Percent predicted forced expiratory volume in the first second (FEV1\%) and percent predicted forced vital capacity (FVC\%) are the primary ventilatory parameters reported herein. The restriction was defined as total lung capacity below the 5th percentile and normal FEV1/FVC ratio (the American Thoracic Society guidelines for restriction (Pellegrino et al. 2005).

\section{Liver Function Tests:}


Serum glutamic oxaloacetic transaminase (SGOT), glutamic pyruvic transaminase (SGPT), total proteins, serum albumin and globulin were measured. Blood samples were analyzed by Beckman AU480 automated chemistry analyzer, USA.

IV. Kidney Function Tests: e.g. serum urea and creatinine were estimated (Fabiny and Ertingshausen, 1971).

V. Complete Blood Count (CBC): EDTA samples were analyzed within 24 hours by SIEMENS ADVIA 2120i HEMATOLOGY ANALYZER, Germany

VI: Serum acetylecholine esterase (AChE) assessment

Five milliliters of blood were drawn from all participants and serum AChE was determined according to (Weber, 1966) using standard kits (Test-combination Boehringer Mannheim GmbH Diagnostica). Serum AChE was selected because it is a better short-term indicator of cholinesterase inhibition than red blood corpuscles (RBCs) AChE due to its more rapid response to exposure; used as an indicator of recent, acute exposure to cholinesterase inhibiting pesticides. Also, because the primary pesticide being applied is chlorpyrifos, which has a preferentially inhibiting effect on serum AChE rather than RBCs AChE (Abdel-Rasoul et al., 2008).

\section{Data management:}

Data were analyzed using IBM SPSS version 22 (SPSS Inc., Chicago, Illinois, USA). Z test was used to test for significance of proportions. For quantitative data, the comparison between two groups was conducted using the student t-test and paired t-test for the same group at two occasions (pre-season and post-season). The Pearson correlation test and t-test for correlation coefficient $(r)$ were used to examine the association between two quantitative variables. P-values less than 0.05 were considered statistically significant

\section{Results:}

There is a non-significant difference between exposed and control groups regarding socio-demographic data (age, income and education level) and BMI ( $P>0.05)$ as shown in Table 1. 
Table 1

Sociodemographic and anthropometric data of exposed and control groups.

\begin{tabular}{|c|c|c|c|c|c|c|}
\hline \multirow[t]{3}{*}{ Sociodemographic \& anthropometric data } & \multicolumn{4}{|c|}{ Studied groups } & \multirow[t]{3}{*}{ Test of significance } & \multirow[t]{3}{*}{ P value } \\
\hline & \multicolumn{2}{|c|}{$\begin{array}{l}\text { Exposed } \\
(n=100)\end{array}$} & \multicolumn{2}{|c|}{$\begin{array}{l}\text { Controls } \\
(n=50)\end{array}$} & & \\
\hline & No. & $\%$ & No. & $\%$ & & \\
\hline Age (years): & \multicolumn{2}{|c|}{$14.25 \pm 1.88$} & \multicolumn{2}{|c|}{$13.80 \pm 2.09$} & t-test $=$ & 0.19 \\
\hline - MeanェSD & \multicolumn{2}{|c|}{$9-18$} & \multicolumn{2}{|c|}{$10-18$} & 1.29 & \\
\hline \multicolumn{7}{|l|}{ - Range } \\
\hline Education: & 7 & 7.0 & 5 & 10.0 & $\mathrm{Z}$ test & 0.75 \\
\hline - Primary & 53 & 53.0 & 24 & 48.0 & 0.32 & 0.68 \\
\hline - Preparatory & 40 & 40.0 & 21 & 42.0 & 0.40 & 0.95 \\
\hline \multicolumn{5}{|l|}{ - Secondary } & \multicolumn{2}{|l|}{0.06} \\
\hline SEL *: & 59 & 59.0 & 24 & 48.0 & Z test & 0.27 \\
\hline - Low $(<5)$ & 24 & 24.0 & 18 & 36.0 & 1.10 & 0.17 \\
\hline - Moderate (5-8) & 17 & 17.0 & 8 & 16.0 & 1.35 & 0.94 \\
\hline \multicolumn{5}{|l|}{ - High (9-12) } & \multicolumn{2}{|l|}{0.08} \\
\hline Weight (Kg) & \multicolumn{2}{|c|}{$50.68 \pm 13.72$} & \multicolumn{2}{|c|}{$51.08 \pm 12.41$} & t-test $=$ & 0.86 \\
\hline - Mean $\pm S D$ & \multicolumn{2}{|c|}{$32-69$} & \multicolumn{2}{|c|}{$30-71$} & 0.17 & \\
\hline \multicolumn{7}{|l|}{ - Range } \\
\hline Height (cm) & \multicolumn{2}{|c|}{$155.36 \pm 7.35$} & \multicolumn{2}{|c|}{$156.69 \pm 8.43$} & t-test $=$ & 0.32 \\
\hline - Mean士SD & \multicolumn{2}{|c|}{$132-169$} & \multicolumn{2}{|c|}{$130-165$} & 0.98 & \\
\hline \multicolumn{7}{|l|}{ - Range } \\
\hline BMI $\left(\mathrm{kg} / \mathrm{m}^{2}\right)^{\star *}$ & \multicolumn{2}{|c|}{$20.19 \pm 2.66$} & \multicolumn{2}{|c|}{$19.59 \pm 2.57$} & t-test = & 0.41 \\
\hline - MeanıSD & \multicolumn{2}{|c|}{$13.60-22.50$} & \multicolumn{2}{|c|}{$12.80-22.30$} & 0.83 & \\
\hline - Range & & & & & & \\
\hline
\end{tabular}

Table 2, Fig. 2 and Fig. 3 show a statistically significant higher prevalence of cough, wheezes, dyspnea and asthma among the exposed group (post season) than each of pre-season and the control group $(\mathrm{P}<0.05)$.

Table (2): Prevalence of respiratory manifestations among exposed (pre and post season) and control groups.

a Comparison between exposed group (pre-season) and control group. 


\begin{tabular}{|c|c|c|c|c|c|c|c|c|c|c|}
\hline \multirow{5}{*}{$\begin{array}{l}\text { Respiratory } \\
\text { manifestations }\end{array}$} & \multicolumn{8}{|c|}{ Studied groups } & \multirow[t]{5}{*}{$Z$ test** } & \multirow[t]{5}{*}{$P$ value } \\
\hline & \multirow{2}{*}{\multicolumn{6}{|c|}{$\begin{array}{l}\text { Exposed } \\
(n=100)\end{array}$}} & \multirow{2}{*}{\multicolumn{2}{|c|}{$\begin{array}{l}\text { Controls } \\
(n=50)\end{array}$}} & & \\
\hline & & & & & & & & & & \\
\hline & \multicolumn{2}{|c|}{$\begin{array}{l}\text { Pre- } \\
\text { season }\end{array}$} & \multicolumn{2}{|c|}{ Post season } & \multirow[t]{2}{*}{ Z test } & \multirow[t]{2}{*}{$P$ value } & \multirow[t]{2}{*}{ No. } & \multirow[t]{2}{*}{$\%$} & & \\
\hline & No. & $\%$ & No. & $\%$ & & & & & & \\
\hline \multirow[t]{2}{*}{ Rhinitis } & 4 & 4.0 & 11 & 11.0 & 3.53 & 0.04 & 3 & 6.0 & $0.14^{a}$ & $0.89^{a}$ \\
\hline & & & & & & & & & $1.13^{b}$ & $0.26^{b}$ \\
\hline \multirow[t]{2}{*}{ Cough } & 6 & 6.0 & 24 & 24.0 & 12.71 & $<0.001$ & 2 & 4.0 & $0.27^{a}$ & $0.68^{a}$ \\
\hline & & & & & & & & & $1.83^{b}$ & $0.03^{b}$ \\
\hline \multirow[t]{2}{*}{ Expectoration } & 9 & 9.0 & 13 & 13.0 & 0.46 & 0.49 & 4 & 8.0 & $0.10^{a}$ & $0.91^{a}$ \\
\hline & & & & & & & & & $0.27^{b}$ & $0.78^{b}$ \\
\hline \multirow[t]{2}{*}{ Wheezes } & 7 & 7.0 & 27 & 27.0 & 12.79 & 0.003 & 2 & 4.0 & $0.36^{a}$ & $0.72^{a}$ \\
\hline & & & & & & & & & $2.81^{b}$ & $0.004^{b}$ \\
\hline \multirow[t]{2}{*}{ Dyspnea } & 6 & 6.0 & 26 & 26.0 & 14.88 & $<0.001$ & 2 & 4.0 & $0.27^{a}$ & $0.68^{a}$ \\
\hline & & & & & & & & & $3.04^{b}$ & $0.002^{b}$ \\
\hline \multirow[t]{2}{*}{ Chest pain } & 3 & 3.0 & 6 & 6.0 & 1.05 & 0.31 & 1 & 2.0 & $0.18^{a}$ & $0.85^{a}$ \\
\hline & & & & & & & & & $0.27^{b}$ & $0.68^{b}$ \\
\hline \multirow[t]{2}{*}{ Asthma } & 2 & 2.0 & 7 & 7.0 & 1.86 & 0.17 & 0 & 0.0 & $0.25^{a}$ & $0.80^{a}$ \\
\hline & & & & & & & & & $3.68^{\mathrm{b}}$ & $0.04^{\mathrm{b}}$ \\
\hline
\end{tabular}

${ }^{\mathrm{b}}$ Comparison between exposed group (post season) and control group.

Table 3 shows that the prevalence of respiratory manifestations (cough, wheezes, dyspnea and asthma) among the exposed group was significantly increased with the decrease in AChE level $(P<0.05)$. 
Table 3

Mean \pm SD of Acetyl Cholinesterase level among the exposed group according to presence or absence of respiratory manifestations at post season.

\begin{tabular}{|c|c|c|c|c|}
\hline \multirow[t]{2}{*}{ Respiratory manifestations } & \multirow[t]{2}{*}{ No. } & Acetyl cholinesterase (AChE) level (IU/L) & \multirow[t]{2}{*}{ t-test } & \multirow[t]{2}{*}{$P$ value } \\
\hline & & Mean $\pm S D$ & & \\
\hline Rhinitis: & 11 & $229.66 \pm 21.98$ & 1.52 & 0.13 \\
\hline Present & 89 & $241.47 \pm 24.62$ & & \\
\hline \multicolumn{5}{|l|}{ Absent } \\
\hline Cough: & 24 & $229.36 \pm 17.83$ & 2.38 & 0.02 \\
\hline Present & 76 & $241.64 \pm 23.17$ & & \\
\hline \multicolumn{5}{|l|}{ Absent } \\
\hline Expectoration: & 13 & $235.20 \pm 16.99$ & 0.90 & 0.37 \\
\hline Present & 87 & $241.53 \pm 24.52$ & & \\
\hline \multicolumn{5}{|l|}{ Absent } \\
\hline Wheezes: & 27 & $228.45 \pm 26.18$ & 2.63 & 0.01 \\
\hline Present & 73 & $243.06 \pm 24.15$ & & \\
\hline \multicolumn{5}{|l|}{ Absent } \\
\hline Dyspnea: & 26 & $227.14 \pm 30.59$ & 2.67 & 0.009 \\
\hline Present & 74 & $242.94 \pm 24.15$ & & \\
\hline \multicolumn{5}{|l|}{ Absent } \\
\hline Chest pain: & 6 & $230.92 \pm 19.92$ & 1.02 & 0.31 \\
\hline Present & 94 & $241.49 \pm 24.95$ & & \\
\hline \multicolumn{5}{|l|}{ Absent } \\
\hline Asthma: & 7 & $228.52 \pm 23.79$ & 1.86 & 0.04 \\
\hline Present & 93 & $242.90 \pm 19.39$ & & \\
\hline Absent & & & & \\
\hline
\end{tabular}

There are statistically significant lower mean values of spirometric measurements (FEV1\%, FEV1/FVC\%, FEF25-75\% and PEF \%) among the exposed group (post season) than each of pre-season and the control group $(P<0.05)$ as shown in Table 4. 
Table 4

Mean \pm SD of spirometric measurements for the exposed and control groups

\begin{tabular}{|c|c|c|c|c|c|c|c|}
\hline \multirow{3}{*}{$\begin{array}{l}\text { Spirometric } \\
\text { measurements } \\
\text { Pre-season }\end{array}$} & \multicolumn{5}{|l|}{ Studied groups } & \multirow[t]{4}{*}{ t-test } & \multirow{4}{*}{$\begin{array}{l}P \\
\text { value }\end{array}$} \\
\hline & \multicolumn{4}{|c|}{ Exposed $(n=100)$} & \multirow[t]{3}{*}{$\begin{array}{l}\text { Controls } \\
(n=50)\end{array}$} & & \\
\hline & Post season & \multirow{2}{*}{$\begin{array}{l}\text { Paired } \\
\text { t-test }\end{array}$} & \multirow{2}{*}{$\begin{array}{l}P \\
\text { value }\end{array}$} & \multirow[t]{2}{*}{ Mean $\pm S D$} & & & \\
\hline Mean $\pm S D$ & Mean \pm SD & & & & & & \\
\hline \multirow[t]{2}{*}{$\mathrm{FVC} \%$} & $91.73 \pm 19.81$ & $88.53 \pm 22.71$ & 1.06 & 0.28 & $93.90 \pm 11.07$ & $0.72^{\mathrm{a}}$ & $0.47^{\mathrm{a}}$ \\
\hline & & & & & & $1.51^{b}$ & $0.13^{b}$ \\
\hline \multirow[t]{2}{*}{$\mathrm{FEV}_{1} \%$} & $102.44 \pm 19.12$ & $99.14 \pm 17.59$ & 1.57 & 0.04 & $104.18 \pm 11.54$ & $0.59^{\mathrm{a}}$ & $0.55^{\mathrm{a}}$ \\
\hline & & & & & & $1.66^{\mathrm{b}}$ & $0.04^{b}$ \\
\hline \multirow{2}{*}{$\begin{array}{l}\mathrm{FEV}_{1} / \\
\mathrm{FVC} \%\end{array}$} & $101.27 \pm 22.48$ & $96.73 \pm 25.88$ & 1.62 & 0.03 & $103.81 \pm 13.09$ & $0.74^{\mathrm{a}}$ & $0.46^{\mathrm{a}}$ \\
\hline & & & & & & $2.63^{b}$ & $0.009^{b}$ \\
\hline \multirow[t]{2}{*}{$\mathrm{FEF}_{25-75} \%$} & $99.13 \pm 17.64$ & $91.17 \pm 23.96$ & 2.68 & 0.008 & $104.17 \pm 19.72$ & $1.59^{\mathrm{a}}$ & $0.11^{\mathrm{a}}$ \\
\hline & & & & & & $3.65^{b}$ & $0.003^{b}$ \\
\hline \multirow[t]{2}{*}{ PEF\% } & $59.16 \pm 18.91$ & $55.07 \pm 18.23$ & 1.89 & 0.02 & $62.05 \pm 16.95$ & $1.02^{\mathrm{a}}$ & $0.34^{\mathrm{a}}$ \\
\hline & & & & & & $2.33^{b}$ & $0.02^{b}$ \\
\hline \multicolumn{8}{|c|}{ a Comparison between exposed group (pre-season) and control group. } \\
\hline
\end{tabular}

Table 5 and Fig. 1 show that means of Acetylcholinesterase (AChE), total protein, albumin and Albumin/Globulin $(\mathrm{A} / \mathrm{G})$ ratio levels were significantly lower, while GPT, GOT, and globulin, blood urea and serum creatinine were significantly higher among the exposed group (post season) than each of pre-season and the control group $(P<0.05)$. 
Table 5

Mean \pm SD of Acetylcholinesterase (AChE) level, liver and kidney function tests among the exposed and control groups.

\begin{tabular}{|c|c|c|c|c|c|c|c|}
\hline \multirow{3}{*}{$\begin{array}{l}\text { Studied } \\
\text { Parameters } \\
\text { Pre-season }\end{array}$} & \multicolumn{5}{|l|}{ Studied groups } & \multirow[t]{4}{*}{ t-test } & \multirow[t]{4}{*}{$P$ value } \\
\hline & \multicolumn{4}{|c|}{ Exposed $(n=100)$} & \multirow{3}{*}{$\begin{array}{l}\text { Controls } \\
(n=50)\end{array}$} & & \\
\hline & Post season & \multirow{2}{*}{$\begin{array}{l}\text { Paired } \\
\text { t test }\end{array}$} & \multirow{2}{*}{$\begin{array}{l}P \\
\text { value }\end{array}$} & \multirow[t]{2}{*}{ Mean $\pm S D$} & & & \\
\hline Mean $\pm S D$ & Mean $\pm S D$ & & & & & & \\
\hline \multirow[t]{2}{*}{ AChE (IU/L) } & $299.60 \pm 42.87$ & $238.49 \pm 23.83$ & 12.66 & $<0.001$ & $302.70 \pm 36.54$ & $0.44^{\mathrm{a}}$ & $0.66^{a}$ \\
\hline & & & & & & $7.73^{\mathrm{b}}$ & $<0.001^{b}$ \\
\hline \multirow[t]{2}{*}{ SGPT (u/l) } & $22.51 \pm 3.29$ & $24.65 \pm 3.72$ & 4.31 & 0.004 & $21.80 \pm 5.08$ & $1.03^{\mathrm{a}}$ & $0.30^{\mathrm{a}}$ \\
\hline & & & & & & $4.59^{b}$ & $0.007^{b}$ \\
\hline \multirow[t]{2}{*}{ SGOT (u/l) } & $26.09 \pm 4.12$ & $28.22 \pm 5.43$ & 3.12 & 0.002 & $27.11 \pm 8.87$ & $0.96^{a}$ & $0.33^{a}$ \\
\hline & & & & & & $2.56^{\mathrm{b}}$ & $0.01^{b}$ \\
\hline \multirow[t]{2}{*}{$\operatorname{ALP}(\mathrm{u} / \mathrm{I})$} & $169.11 \pm 49.33$ & $171.02 \pm 55.41$ & 0.26 & 0.79 & $154.91 \pm 55.92$ & $1.59^{a}$ & $0.11^{a}$ \\
\hline & & & & & & $1.84^{\mathrm{b}}$ & $0.04^{b}$ \\
\hline \multirow[t]{2}{*}{$\begin{array}{l}\text { Total protein } \\
(\mathrm{gm} / \mathrm{l})\end{array}$} & $7.09 \pm 1.03$ & $6.74 \pm 1.13$ & 2.29 & 0.02 & $7.37 \pm 1.51$ & $1.34^{\mathrm{a}}$ & $0.18^{a}$ \\
\hline & & & & & & $3.31^{\mathrm{b}}$ & $0.001^{b}$ \\
\hline \multirow[t]{2}{*}{ Albumin (gm/l) } & $3.94 \pm 0.59$ & $3.59 \pm 0.55$ & 4.34 & 0.003 & $4.07 \pm 0.52$ & $1.32^{\mathrm{a}}$ & $0.19^{a}$ \\
\hline & & & & & & $5.46^{\mathrm{b}}$ & $<0.001^{b}$ \\
\hline \multirow[t]{2}{*}{ Globulin (gm/l) } & $1.93 \pm 0.50$ & $1.99 \pm 0.51$ & 0.84 & 0.40 & $1.87 \pm 0.57$ & $0.66^{a}$ & $0.51^{\mathrm{a}}$ \\
\hline & & & & & & $3.33^{\mathrm{b}}$ & $0.003^{b}$ \\
\hline \multirow[t]{2}{*}{$\begin{array}{l}\text { Albumin/Globulin } \\
\text { (A/G) ratio }\end{array}$} & $2.16 \pm 0.41$ & $1.61 \pm 0.41$ & 9.49 & $<0.001$ & $2.29 \pm 0.89$ & $1.23^{\mathrm{a}}$ & $0.22^{a}$ \\
\hline & & & & & & $6.41^{\mathrm{b}}$ & $<0.001^{b}$ \\
\hline \multirow[t]{2}{*}{$\begin{array}{l}\text { Blood urea } \\
(\mathrm{mg} / \mathrm{dl})\end{array}$} & $24.95 \pm 5.78$ & $28.27 \pm 4.72$ & 4.45 & 0.004 & $24.67 \pm 6.41$ & $0.27^{a}$ & $0.78^{a}$ \\
\hline & & & & & & $4.56^{\mathrm{b}}$ & $0.002^{b}$ \\
\hline \multirow[t]{2}{*}{$\begin{array}{l}\text { S. creatinine } \\
(\mathrm{mg} / \mathrm{dl})\end{array}$} & $0.84 \pm 0.12$ & $1.02 \pm 0.08$ & 12.48 & $<0.001$ & $0.84 \pm 0.13$ & $0.41^{a}$ & $0.68^{a}$ \\
\hline & & & & & & $2.74^{\mathrm{b}}$ & $0.006^{b}$ \\
\hline \multicolumn{8}{|c|}{ a Comparison between exposed group (pre-season) and control group } \\
\hline
\end{tabular}


Table 6 shows statistically significant lower measurements of red blood corpuscles (RBCs), Hb and lymphocyte among the exposed group (post-season) than each of pre-season measurements and the control group $(P<0.05)$.

Table 6

Mean \pm SD of CBC parameters among the exposed and control groups.

\begin{tabular}{|c|c|c|c|c|c|c|c|}
\hline \multirow[t]{2}{*}{$\mathrm{CBC}$ parameters } & \multicolumn{5}{|l|}{ Studied groups } & \multirow[t]{2}{*}{ t-test } & \multirow[t]{2}{*}{$P$ value } \\
\hline & \multicolumn{4}{|c|}{ Exposed $(n=100)$} & $\begin{array}{l}\text { Controls } \\
(n=50)\end{array}$ & & \\
\hline Pre-season & Post season & \multirow[t]{2}{*}{ Paired $t$ test } & \multirow{2}{*}{$\begin{array}{l}\mathrm{P} \\
\text { value }\end{array}$} & \multirow[t]{2}{*}{ Mean $\pm S D$} & & & \\
\hline Mean $\pm S D$ & Mean $\pm S D$ & & & & & & \\
\hline \multirow[t]{2}{*}{ RBCs $\left(10^{6} / \mathrm{l}\right)$} & $4.71 \pm 0.37$ & $4.19 \pm 0.31$ & 10.77 & 0.003 & $4.82 \pm 0.42$ & $1.50^{\mathrm{a}}$ & $0.14^{\mathrm{a}}$ \\
\hline & & & & & & $12.85^{\mathrm{b}}$ & $<0.001^{b}$ \\
\hline \multirow[t]{2}{*}{$\begin{array}{l}\text { Hemoglobin } \\
(\mathrm{Hb})(\mathrm{gm} / \mathrm{dl})\end{array}$} & $12.62 \pm 0.98$ & $12.32 \pm 0.65$ & 2.38 & 0.02 & $12.63 \pm 1.01$ & $1.11^{\mathrm{a}}$ & $0.27^{\mathrm{a}}$ \\
\hline & & & & & & $2.48^{\mathrm{b}}$ & $0.01^{\mathrm{b}}$ \\
\hline \multirow{2}{*}{$\begin{array}{l}\text { Leucocytes } \\
\left(10^{3} / \mathrm{mm}^{3}\right)\end{array}$} & $6.69 \pm 2.02$ & $6.83 \pm 2.01$ & 0.49 & 0.62 & $6.48 \pm 2.36$ & $0.55^{\mathrm{a}}$ & $0.58^{\mathrm{a}}$ \\
\hline & & & & & & $1.75^{\mathrm{b}}$ & $0.15^{\mathrm{b}}$ \\
\hline \multirow{2}{*}{$\begin{array}{l}\text { Lymphocytes } \\
\left(10^{3} / \mathrm{dl}\right)\end{array}$} & $3.58 \pm 1.08$ & $2.98 \pm 0.68$ & 4.70 & $<0.001$ & $3.63 \pm 0.71$ & $0.24^{\mathrm{a}}$ & $0.81^{\mathrm{a}}$ \\
\hline & & & & & & $6.67^{b}$ & $0.006^{\mathrm{b}}$ \\
\hline \multirow[t]{2}{*}{$\begin{array}{l}\text { Basophiles } \\
\left(10^{3} / \mathrm{dl}\right)\end{array}$} & $78.71 \pm 20.62$ & $76.33 \pm 23.11$ & 0.77 & 0.44 & $77.89 \pm 23.73$ & $0.22^{\mathrm{a}}$ & $0.83^{a}$ \\
\hline & & & & & & $0.42^{\mathrm{b}}$ & $0.68^{\mathrm{b}}$ \\
\hline \multirow{2}{*}{$\begin{array}{l}\text { Platelets } \\
\left(10^{3} / \mathrm{mm}^{3}\right)\end{array}$} & $249.24 \pm 71.61$ & $244.57 \pm 43.91$ & 0.56 & 0.57 & $254.56 \pm 63.88$ & $0.44^{\mathrm{a}}$ & $0.65^{\mathrm{a}}$ \\
\hline & & & & & & $0.67^{b}$ & $0.50^{\mathrm{b}}$ \\
\hline
\end{tabular}

Table 7 shows that there was a statistically significant positive correlation between AChE level and spirometric measurements for the exposed group (FVC\%, FEV1\%, FEV1/FVC\%, FEF25-75\% and PEF\% ( $r=0.62,0.41,0.55,0.61$ and 0.43; respectively) $(\mathrm{P}<0.05)$. On the opposite side, it shows a negative correlation between AChE and SGPT, SGOT, ALP, globulin, blood urea and serum creatinine for the exposed group reaching a significant level with both SGPT and SGOT $(P<0.05)$. Also, a non-significant positive correlation was found between $A C h E(I U / L)$ and each of total protein, albumin and $A / G$ ratio $(P>0.05)$. At the same time, there was a positive correlation between AChE level and all studied CBC parameters for the exposed group, only basophils showed a statistically significant correlation 
$(P<0.05)$. The values of $R^{2}$ denote a range of percentages for the goodness of the studied variables significantly associated with AChE between $10-38 \%$.

Table 7: Correlation between Acetyl cholinesterase (AChE) level with each of spirometric measurements, liver and kidney function tests and CBC parameters among the exposed group.

\begin{tabular}{|c|c|c|c|c|}
\hline \multirow[t]{2}{*}{ Studied parameters } & \multicolumn{4}{|c|}{ AChE (IU/L) } \\
\hline & $r$ & $\mathrm{R}^{2}$ & t-test* & $P$ value \\
\hline \multicolumn{5}{|c|}{ 1-Spirometric measurements } \\
\hline FVC\% & 0.62 & 0.384 & 7.822 & $<0.005$ \\
\hline $\mathrm{FEV}_{1} \%$ & 0.41 & 0.168 & 4.450 & $<0.005$ \\
\hline $\mathrm{FEV}_{1} / \mathrm{FVC} \%$ & 0.55 & 0.302 & 6.519 & $<0.005$ \\
\hline $\mathrm{FEF}_{25-75} \%$ & 0.61 & 0.372 & 7.620 & $<0.005$ \\
\hline PEF\% & 0.43 & 0.185 & 4.714 & $<0.005$ \\
\hline \multicolumn{5}{|l|}{ 2- Liver \& kidney functions } \\
\hline SGPT (u/l) & -0.27 & 0.073 & 2.775 & $<0.005$ \\
\hline SGOT (u/l) & -0.28 & 0.078 & 2.887 & $<0.005$ \\
\hline $\operatorname{ALP}(u / I)$ & -0.16 & 0.025 & 1.604 & 0.10 \\
\hline Total protein gm/l & 0.03 & 0.001 & 0.297 & 0.50 \\
\hline Albumin (gm/l) & 0.07 & 0.005 & 0.694 & 0.50 \\
\hline Globulin (gm/l) & -0.25 & 0.062 & 2.556 & $<0.01$ \\
\hline $\mathrm{A} / \mathrm{G}$ ratio & 0.17 & 0.029 & 1.707 & 0.50 \\
\hline Blood urea $(\mathrm{mg} / \mathrm{dl})$ & -0.19 & 0.036 & 1.915 & $<0.05$ \\
\hline Serum creatinine $(\mathrm{mg} / \mathrm{dl})$ & -0.05 & 0.002 & 0.495 & 0.50 \\
\hline \multicolumn{5}{|l|}{ 3- CBC Parameters } \\
\hline RBCs $\left(10^{6} / \mathrm{I}\right)$ & 0.17 & 0.029 & 1.707 & 0.50 \\
\hline Hemoglobin (gm/dl) & 0.06 & 0.002 & 0.595 & 0.50 \\
\hline Leucocytes $\left(10^{3} / \mathrm{mm}^{3}\right)$ & 0.07 & 0.005 & 0.694 & 0.50 \\
\hline Lymphocytes $\left(10^{3} / \mathrm{dl}\right)$ & 0.13 & 0.027 & 1.297 & 0.20 \\
\hline Basophils $\left(10^{3} / \mathrm{dl}\right)$ & 0.23 & 0.152 & 2.339 & $<0.02$ \\
\hline Platelets $\left(10^{3} / \mathrm{mm}^{3}\right)$ & 0.16 & 0.026 & 1.604 & 0.10 \\
\hline
\end{tabular}




\section{Discussion:}

The exposed and control groups were matched for demographic and anthropometric measurements e.g age (in years), level of education, weight $(\mathrm{kgm})$, height $(\mathrm{cm})$, and body mass index (BMI), they didn't show any statistically significant difference $(P>0.05$, Table 1).

This study revealed a significantly higher prevalence of health effects in adolescent females environmentally exposed to pesticides compared to the control group. Exposed participants had lower. AChE activity levels, impaired ventilatory functions and more prevalent respiratory manifestations.

The exposed adolescent females reported a significantly higher prevalence of respiratory manifestations as cough, wheezes, dyspnea, asthma (24\%, $27 \%, 26 \%$ and $7 \%$; respectively) compared to the control participants (10\%, 6\%, 4\%, $6 \%$ and $0 \%$; respectively) and also in post exposure than pre exposure season $(P<0.05$, Table 2, Figs. $2-3)$. Moreover, exposed adolescent females positive for these manifestations had significantly lower AChE levels than negative ones $(\mathrm{P}<0.05$, Table 3$)$.. These findings coincide with those of previous studies conducted on agricultural workers and pesticide applicators (Ohayo-Mitoko, 2000). Also, pesticide use in the kitchen or dining rooms was associated with an increased prevalence of wheezes among children under 18 years of age in the United States (Hoppin et al., 2002). A study on Canadian farmers reported a significant risk for asthma with use of carbamate insecticides $(\mathrm{OR}=1.8 ; 95 \% \mathrm{Cl} 1.1$ - 3.1) compared to non-asthmatic farmers (Senthilselvan et al. 1992). This is further evidenced by lung dysfunction indicated by lower pulmonary function values as observed in asthmatic farmers exposed to. carbofuran, methomyl, and carbaryl pesticides (Mamane et al. 2015).

Additionally, the exposed adolescent females showed statistically significant lower spirometric measurements than the control ones. It was found that mean values of spirometric measurements as (FEV1\%, FEV1/FVC\%, FEF25-75\% and PEF \%) were significantly lower in the exposed group $(99.14 \pm 17.59,96.73 \pm 25.88,91.17 \pm 23.96$ and $55.07 \pm 18.23$; respectively) than their controls $(104.18 \pm 11.54,103.81 \pm 13.09,104.17 \pm 19.72$ and $62.05 \pm 16.95$; respectively) $(\mathrm{P}<0.05$, Table 4$)$. These findings are consistent with previous studies of spirometry and OP exposure in adults as Callahan et al. (2014) who found significantly lower FEV1\% and FVC\% among applicators compared to non-applicators. Also, these findings are supported by the results of Peiris-John et al. (2005) who found that lowered FVC\% and OP exposure were associated in 25 occupationally exposed Sri Lankan farmers and 22 fishermen who lived within a $25 \mathrm{~km}$ radius of fields where OPs were sprayed than non-exposed controls. Similarly, Zhu et al. (2015) found that pesticide applicators aged from 15-24 years had lower spirometric measurements than non-applicators with the same age. However, among 89 greenhouse workers and 25 non-spraying controls in Spain spirometric measurements and exposure to OPs were not associated; OPs exposure was defined as a depression of more than $25 \%$ in plasma cholinesterase or $15 \%$ depression in AChE levels (Hernandez et al. 2008).

The association berween increased prevalence of respiratory symptms and the decreased measurenents could due to direct allergic effects from inhalation of OPs compounds or from the nicotinic effect of ACh due to inhibition of AChE.

This study showed statistically significant lower means of AChE level, total protein, albumin, albumin/globulin (A/G) ratio in the exposed at post exposure $(238.49 \pm 23.83 \mathrm{IU} / \mathrm{L}, 6.74 \pm 1.13 \mathrm{gm} / \mathrm{l}, 3.59 \pm 0.55 \mathrm{gm} / \mathrm{l}$ and $1.61 \pm 0.04$; respectively) than either the controls $(302.70 \pm 36.54 \mathrm{IU} / \mathrm{L}, 7.37 \pm 1.51 \mathrm{gm} / \mathrm{l}, 4.07 \pm 0.53 \mathrm{gm} / \mathrm{l}$ and 2.29 \pm 0.89 ; 
respectively) or the exposed at pre exposure season $(299.60 \pm 42.87 \mathrm{IU} / \mathrm{L}, 7.09 \pm 1.03 \mathrm{gm} / \mathrm{l}, 3.94 \pm 0.59 \mathrm{gm} / \mathrm{l}$ and $2.16 \pm 0.41$; respectively $(P<0.05$, Fig. 1 , Table 5$)$.

On the other hand, statistically significant higher means of SGPT, SGOT, ALP, globulin, urea, and creatinine were found in the exposed group post season $(24.65 \pm 3.72 \mathrm{u} / \mathrm{l}, 28.22 \pm 5.43 \mathrm{u} / \mathrm{l}, 169.11 \pm 49.33 \mathrm{u} / \mathrm{l}, 1.99 \pm 0.51 \mathrm{gm} / \mathrm{l}$, $28.27 \pm 4.72 \mathrm{mg} / \mathrm{dl}$, and $1.02 \pm 0.08 \mathrm{mg} / \mathrm{dl}$; respectively) than either of the controls $(20.61 \pm 7.07 \mathrm{u} / \mathrm{l}, 25.61 \pm 6.73 \mathrm{u} / \mathrm{l}$, $154.71 \pm 55.92 \mathrm{u} / \mathrm{l}, 1.68 \pm 0.59 \mathrm{gm} / \mathrm{l}, 24.40 \pm 5.25 \mathrm{mg} / \mathrm{dl}$, and $0.95 \pm 0.23 \mathrm{mg} / \mathrm{dl}$; respectively) or the exposed in the pre exposure season $(22.51 \pm 3.29 \mathrm{u} / \mathrm{l}, 26.06 \pm 4.12 \mathrm{u} / \mathrm{l}, 169.11 \pm 48.33 \mathrm{u} / \mathrm{l}, 1.93 \pm 0.50 \mathrm{gm} / \mathrm{l}, 24.95 \pm 5.78 \mathrm{mg} / \mathrm{dl}$ and $0.84 \pm 0.12 \mathrm{mg} / \mathrm{dl}$; respectively) $(\mathrm{P}<0.05$, Table 5$)$.

The statistically significant increase in SGOT and SGPT for the studied adolescent females agrees with other several studies that reported the deterioration of liver enzymes in association with exposure to pesticides (Farahat et al., 2003; Jørs et al., 2006 and Mansour and Mossa, 2009). Also, these results are in agreement with (El-Sobky et al., 1994) who found that there was a statistically significant increase in SGOT ( $\leq 42$ vs. $>42$ U/L) and SGPT ( $\leq 60$ vs. $>60 \mathrm{U} / \mathrm{L}$ ) at the end of cotton ginning season (1991-1992) (31\% and 30\%; respectively) for exposed 100 participants compared to 50 controls ( $12 \%$ and $12 \%$; respectively).

Also, SGPT, SGOT, ALP, total plasma proteins, albumin, globulin, and albumin/globulin ratio were used to assess liver function, pesticides decrease the total protein and albumin as a result of decrease synthesis of albumin in liver, and increase globulin (Barr et al., 2006). Altered liver enzyme activities have been reported among pesticide workers exposed to OP pesticides alone or in combination with organochlorine or other pesticides (Kamel et al., 2003).

The relatively elevated liver enzymes among exposed than controls elicited in the present study may reflect the hepatic effect of long term exposure to OP pesticides. This assumption was reflected on the chronic hepatocellular biochemical indicators, low protein and low albumin in the exposed than the control participants and could be documented by the statistically significant correlation between duration of exposure and both ALT and alkaline phosphatase. It may be due to that the effect of chronic exposure to pesticides that could result in acute or subacute liver insult (Deziel et al., 2015).

Liver injury would result in the delay or failure of the detoxification mechanisms with consequent earlier development of cumulative pesticide effects and hence a vicious cycle (Rani et al.; 2017). It may be emphasized that hepatic susceptibility to pesticides clinically and biochemically manifested to be mostly related to protein deprivation (Andreotti et al., 2009).

The oxidative damage of liver cells is promoted by exposure to OPs by enhancing peroxidation of membrane lipids marker changes in the overall histoarchitecture of liver in response to OPs,. This might be a result of toxic effects initiated by the production of reactive oxygen species causing destruction to the various membrane components of the cell (Mansour and Mossa, 2009).

The present significant increase in the mean measurements of blood urea and serum creatiine among the environmentally pesticides' exposed adolescent females $(P<0.05$, Table 5$)$ agrees with that obtained by (Khan et al., 2005). Quandt et al., 2010 also found that among the 100 rice farmers in their sample, 23 farmers had abnormal levels of blood urea nitrogen (BUN), 22 of which had BUN values exceeding the upper boundary for the normal population. Similarly, Cavari et al., 2013 proposed that OP effects on the renal system could be due to direct parenchymal intoxication, secondary to hemodynamic instability or seizure-induced rhabdomyolysis (Yardan et al., 2013 and Mohamed et al., 2016). 
On studying the hematological disorders among adolescent females environmentally exposed to pesticides, a statistically significant decrease in means of RBCs a, $\mathrm{Hb}$ and lymphocytes measurements was found among the exposed females in post exposure season $\left(4.19 \pm 0.3110^{6} / \mathrm{l}, 12.322 \pm 0.65 \mathrm{gm} / \mathrm{dl}\right.$ and $2.98 \pm 0.6810^{3} / \mathrm{dl}$; respectively) than either of the controls $\left(4.82 \pm 0.4210^{6} / \mathrm{l}, 12.63 \pm 1.01 \mathrm{gm} / \mathrm{dl}\right.$ and $3.63 \pm 0.7110^{3} / \mathrm{dl}$; respectively) or exposed in pre exposure season $\left(4.71 \pm 0.3710^{6} / \mathrm{l}, 12.62 \pm 0.98 \mathrm{gm} / \mathrm{dl}\right.$ and $3.58 \pm 1.0810^{3} / \mathrm{dl}$; respectively) $(\mathrm{P}<0.05$, Table 6). Decreased RBCs count and hemoglobin may be attributed either to the fact that OP pesticides affect dietary intake (Leach, 2014) or due to the effect of pesticides on the bone marrow (Barr et al., 2006). Another possible mechanism is binding of organophosphate pesticides on iron, followed by a lack of incorporation of iron in hemoglobin (Abu Mourad, 2005).

Moreover, these results are in concordance with those of Mansour and Mossa, 2009 who revealed that most of the hematological, renal and liver indices were affected, where there were significantly lower values of RBC's count, hemoglobin, lymphocytes count, total protein, albumin and A/G ratio in the exposed group (4.05 $\pm 0.24 \mathrm{ml} / \mathrm{l}, 12.37 \pm$ $1.19 \mathrm{gm} / \mathrm{dl}, 2.35 \pm 0.7610^{3} / \mathrm{dl}, 6.2 \pm 0.88 \mathrm{gm} / \mathrm{l}, 3.45 \pm 0.44 \mathrm{gm} / \mathrm{dl}$ and $1.56 \pm 0.42$; respectively) compared to the control group $\left(4.78 \pm 0.6 \mathrm{ml} / \mathrm{l}, 13.26 \pm 1.04 \mathrm{gm} / \mathrm{dl}, 3.02 \pm 0.8610^{3} / \mathrm{dl}, 7.54 \pm 1.13 \mathrm{gm} / \mathrm{l}, 4.12 \pm 0.5 \mathrm{gm} / \mathrm{dl}\right.$ and $2.46 \pm$ 0.9 ; respectively).

The relationship between the exposure to pesticides and decrement in the different spirometric measurements is confirmed by the presence of significant positive correlation of AChE levels with values of FVC\%, FEV1\%, FEV1/FVC\%, FEF25-75\% and PEF \% ( $r=0.62,0.41,0.55,0.61$ and 0.43 ; respectively) and $\left(R^{2}=0.231,0.168,0.302\right.$, 0.372 and 0.185$)(P<0.05$, Table 7). These results are consistent with the study of Chakraborty et al. (2009) who reported that in Indian agricultural workers the inhibition of AChE more than $50 \%$ was associated with increased reporting of respiratory symptoms and reduced lung function (13.6\% lower mean FVC, and 15.6\% lower mean FEV1) than non-agricultural workers..

As mentioned above, the current results showed a significantly lower level of AChE in the exposed adolescent females $(238.49 \pm 23.83 \mathrm{IU} / \mathrm{L})$ compared to the control ones $(303.35 \pm 78.54 \mathrm{IU} / \mathrm{L})(\mathrm{P}<0.05$, Fig. 1 - Table: 5$)$. There are several studies that reported AChE level showing a consistent significant association between exposure to pesticides and AChE inhibition in farm workers. All studies stated that AChE was significantly lower in the exposed participants than the controls (Clayton et al., 2003, Abdel-Rasoul et al., 2008; and Khan et al., 2010). Jørs et al., 2006. reported a mean ChE activity of $7.11 \mathrm{kU} / \mathrm{L}$ for those who have sprayed with OPs compared to a mean ChE of $8.03 \mathrm{kU} / \mathrm{L}$ for those who have not and ChE activity of $8.03 \mathrm{kU} / \mathrm{L}$ for those who have not sprayed compared to a ChE activity of $7.60 \mathrm{kU} / \mathrm{L}$ for those who have sprayed from 1-3 times and a ChE activity of $7.12 \mathrm{kU} / \mathrm{L}$ for those who have sprayed $>3$ times. Moreover, there was a statistically significant negative correlation between levels of AChE and laboratory indices of SGPT ( $r=-0.27$ and $\left.R^{2}=0.073\right)$ and SGOT $\left(r=-0.21\right.$ and $\left.R^{2}=0.044\right)$ and a statidtically significant positive correlation with basophils' count $\left(r=0.23\right.$ and $\left.R^{2}=0.152\right)(P<0.05$, Table 7$)$, which means that there were significant liver effects in addition to clinical and biochemical changes significantly associated with lower levels of AChE (Jørs et al., 2006)

\section{Limitations of the Study:}

To the knowledge of the authors, there is a scanty number of studies and cohorts on the adolescent females environmentally exposed to pesticides. The obtained results could be more evidenced by the inclusion of additional studies. 


\section{Conclusions And Recommendations:}

Pesticides have been linked to numerous adverse health effects that are different in females than in males. Adolescent females living in agricultural areas and from families whose one or more members are pesticides $\Downarrow$ sprayers have statistically significant lower spirometric measurements and higher prevalence of respiratory manifestations; and a statistically significant hepatic, renal and hematological impact compared to the control group. Since adolescents around the world are exposed to OP pesticides, these studies suggest an urgent need to evaluate this potential problem. Future research should focus on the type of health education training considering the perceived benefits and disadvantages while developing plans to decrease health disorders among adolescent females.

\section{Declarations}

Conflicts of interest: None declared.

Funding: not available.

Consent to Participate:

Written informed consents were signed by all participants' parents before being enrolled in the study.

\section{Ethical Approval:}

Medical Ethics Committee at the Menoufia Faculty of Medicine approved the study protocol before starting.

\section{Author Contribution:}

\begin{tabular}{|ll|}
\hline Author's name & Contribution \\
\hline Gaafar Mohamed Abdel-Rasoul & $\begin{array}{l}\text { The owner of the research idea } \\
\text { Study design } \\
\text { Revision of the research article }\end{array}$ \\
\hline Eman Abdel-Azeem Salem & Shared in writing and revision of the manuscript \\
\hline Olfat Mohamed Hendy & Responsible for laboratory investigation \\
\hline Diane Rohlman & Revision of the research article \\
\hline Asmaa Abdel-Rehim Abdel-Latif & Data collection and shared in writing of the manuscript \\
\hline Aziza Saad Elbadry & Shared in writing and revision of the manuscript \\
\hline
\end{tabular}

Consent to Publish: not applicable in our IRB (Ethical Committee, Menoufia University, Faculty of Medicine).

Availability of Data and Materials: available.

\section{References}


1. Abdel-Rasoul G, Abou Salem M, Mechael A, Hendy O, Rohlman D, Ismail A (2008) Effects of occupational pesticide exposure on children applying pesticides.Neuro29(5):833-838

2. Abu Mourad TA (2005) Adverse impact of insecticides on the health of. Palestinian farm workers in the Gaza, Strip A hematologic biomarker study. Int J Occup Environ Health 11:144-149.|

3. Adad L, Andrade H, Kvitko K, Lehmann M, Cavalcante A, Rodrigues R (2015) Occupational exposure of workers to pesticides:Toxicogenetics and susceptibility gene polymorphisms. Gen Molec Bio 38(3): 308-315

4. Andreotti G, Laura E, Freeman B, Hou L, Coble J, Rusiecki J, Alavanja M (2009) Agricultural Pesticide Use and Pancreatic Cancer Risk in the Agricultural Health Study Cohort. Int J Cancer 124(10):2495-2500

5. Baldi I, Lebailly P, Rondeau V (2012) Levels and determinants of pesticide exposure in operators involved in treatment of vineyards: results of the PESTEXPO Study. J Expo Sci Environ Epidemiol 22:593-600

6. Barr DB, Thomas K, Curwin B, Landsittel D, Raymer J, Lu C (2006) Biomonitoring of exposure in farmworker studies. Environ Health Perspect 114:936-942

7. Callahan CL, Al-Batanony M, Ismail AA, Abdel-Rasoul GM, Hendy O, Olson J, Rohlman D, Bonner M (2014) Chlorpyrifos exposure and respiratory health among adolescent agricultural workers. Int J Environ Res Public Health 11:13117-13129

8. Cavari Y, Landau D, Sofer S (2013) Organophosphate poisoning induced acute renal failure. Pediatr Emerg Care 29:646-647

9. Chakraborty S, Mukherjee S, Roychoudhury S, Siddique S, Lahiri T, Ray M (2009) Chronic exposures to cholinesterase-inhibiting pesticides adversely affect respiratory health of agricultural workers in India. J Occup Health 51:488-497

10. Clayton C, Pellizzari E, Whitmore R, Quackenboss J, Sefton K (2003) Distributions, associations, and partial aggregate exposure of pesticides and polynuclear aromatic hydrocarbons in the Minnesota Children's Pesticide Exposure Study (MNCPES). Exp Analys and Environl Epidemio 13:100

11. Deziel N, Laura E, Beane F, Barry I, Rena R, Jane A (2015) Relative Contributions of Agricultural Drift, ParaOccupational, and Residential Use Exposure Pathways to House Dust Pesticide Concentrations. Environ Health Perspect 10:1-39

12. El-Sobky MK, Raouf AA, Abdel-Kareem, Abdel-Rasoul GM, Refaat AM (1994) Hepatic hazards of pesticides residue in cotton ginning industry. MD thesis. Faculty of Medicine, Menoufia University 125-137

13. Fabiny DL, Ertingshausen GT (1971) Automated reaction-rate method for determination of serum creatinine with the Centrifi-Chem. Clin Chem 17:696-700

14. Farahat T, Abdel Rasoul G, Amr M, Shebl M, Farahat F, Anger W (2003) Neurobehavioral effects among workers occupationally exposed to organophosphorus pesticides. Occup and Environ Med 60:279-286

15. Hernandez A, Casado I, Pena G, Gil F, Villanueva E, Pla A (2008) Low level of exposure to pesticides leads to lung dysfunction in occupationally exposed subjects. Inhal Toxicol 20:839-849

16. Hoppin JA, Umbach DM, London SJ, Alavanja MC, Sandler DP (2002) Chemical predictors of wheeze among farmer pesticide applicators in the Agricultural Health Study. Respir Crit Care Med 165(5):683-689

17. Jørs E, Morant RC, Aguilar GC (2006) Occupational pesticide intoxications among farmers in Bolivia: a crosssectional study. Environ Health 4:5-10

18. Kamanyire R, Karalliedde L (2004) Organophosphate toxicity and occupational exposure. Occup Med (Lond) 54(2):69-75 
19. Kamel F, Rowland A, Park L, Anger W, Baird D, Gladen B (2003) Neurobehavioral performance and work experience in Florida farmworkers. Environ Health Perspect 111:1765-1772

20. Khan DA, Shabbir S, Majid M, Naqvi TA, Khan FA (2010) Risk assessment of pesticide exposure on health of Pakistani tobacco farmers. J Expo Sci Environ Epidemiol 20:196-204

21. Khan S, Sobti R, Kataria L (2005) Pesticide-induced alteration in mice hepato-oxidative status and protective of black tea extract. Clin. Chim Acta 358:131-138

22. Leach M (2014) Interpretation of the full blood count in systemic disease--a guide for the physician. J R Coll Physicians Edinb 44(1):36-41

23. Lee F, Chen W, Lin C, Lai C, Wu W, Lin I, Kao C (2015) Organophosphate Poisoning and Subsequent Acute Kidney Injury Risk. Medicine J 94(47):1-8

24. Mamane A, Raherison C, Tessier J, Baldi I, Bouvier G (2015) Environmental exposure to pesticides and respiratory health. Eur Respir Rev 24:462-473

25. Mansour SA, Mossa AT (2009) Lipid peroxidation and oxidative stress in rat erythrocytes induced by chlorpyrifos and the protective effect of zinc. Pestic Biochem Physiol 93:34-39

26. Miller GT (2004) Sustaining the Earth, 6th edition. Thompson Learning, Inc Pacific Grove, California: 9:211-216

27. Mohamed F, Endre Z, Jayamanne S (2016) Mechanisms underlying early rapid increases in creatinine in paraquat poisoning. PLOS One J 10(e0122357):1-11

28. Ohayo-Mitoko GJA, Kromhout H, Simwa JM, Boleij JSM, Heederik D (2000) Self reported symptoms and inhibition of acetylcholinesterase activity among Kenyan agricultural workers. Occup Environ Med 57:195-200

29. Peiris-John RJ, Ruberu DK, Wickremasinghe AR, -der-Hoek W (2005) Low-level exposure to organophosphate pesticides leads to restrictive lung dysfunction. Respir Med 99: 1319-1324.??

30. Pellegrino R, Viegi G, Brusasco V, Crapo RO, Burgos F, Casaburi R, Coates A, van der Grinten CP, Gustafsson P, Hankinson J (2005) Interpretative strategies for lung function tests. Eur Respir J 26:948-968

31. Proskocil BJ, Bruun DA, Lorton JK (2010) Antigen sensitization influences organophosphorus pesticide-induced airway hyperreactivity. Environ Health Perspect 116:381-388

32. Quadrelli S, Roncoroni A, Montiel G (1999) Assessment of respiratory function: Influence of spirometry reference values and normality criteria selection. Respir Med 93:523-535

33. Quandt S, Kucera K, Haynes C, Klein B (2010) Occupational health outcomes for workers in the agriculture, forstry and fishing sector. American Journal of Industrial Mdicine 14(2):125-131

34. Rani M, Shanker U, Jassal V (2017) Recent strategies for removal and degradation of persistent \& toxic organochlorine pesticides using nanoparticles: A review. J Environ Manage 190:208-222

35. Rohlman D, Lasarev M, Anger W, Scherer J, Stupfel J, McCauley L (2007) Neurobehavioral performance of adult and adolescent agricultural workers. Neurotoxicology 28:374-380

36. Senthilselvan A, McDuffie HH, Dosman JA (1992) Association of asthma with use of pesticides. results of a cross-sectional survey of farmers. Am Rev Respir Dis 146:884-887

37. Weber H (1966) Quick and simple ultra-micro-method for the determination of cholinesterase. Dtsch Med Weschr 91:1927-1932

38. Yardan T, Baydin A, Acar E (2013) The role of serum cholinesterase activity and S100B protein in the evaluation of organophosphate poisoning. Hum Exp Toxicol 32:1081-1088

39. Zhu J, Dubois A, Ge Y, Olson JA, Ren X (2015) Application of human haploid cell genetic screening model in identifying the genes required for resistance to environmental toxicants: Chlorpyrifos as a case study. J 
Pharmacol Toxicol Methods 76(154):76-82

\section{Figures}

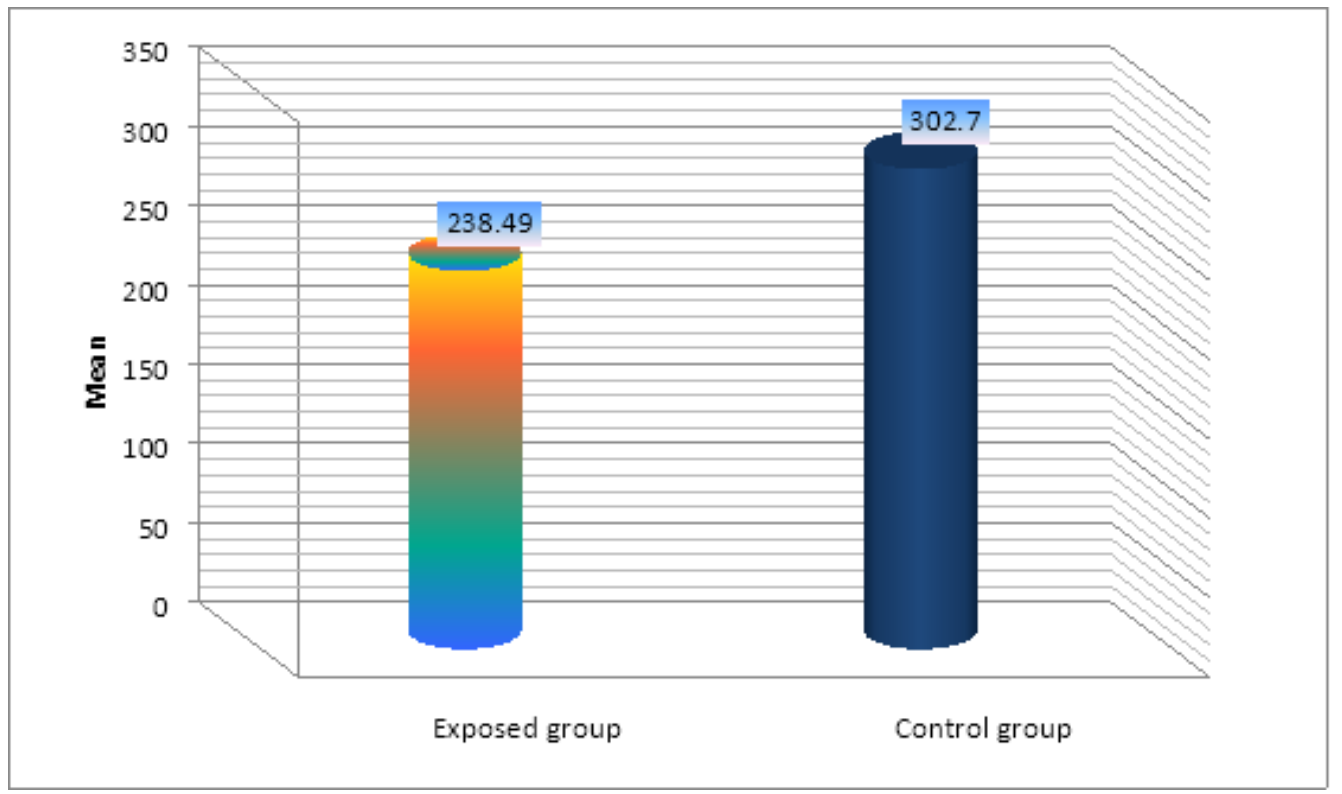

Figure 1

Acetyl cholinesterase (AChE) level among the exposed group (post season) and the control group Figure 1 shows a significant decrease in AChE level among the exposed group (post season) compared to the control group.

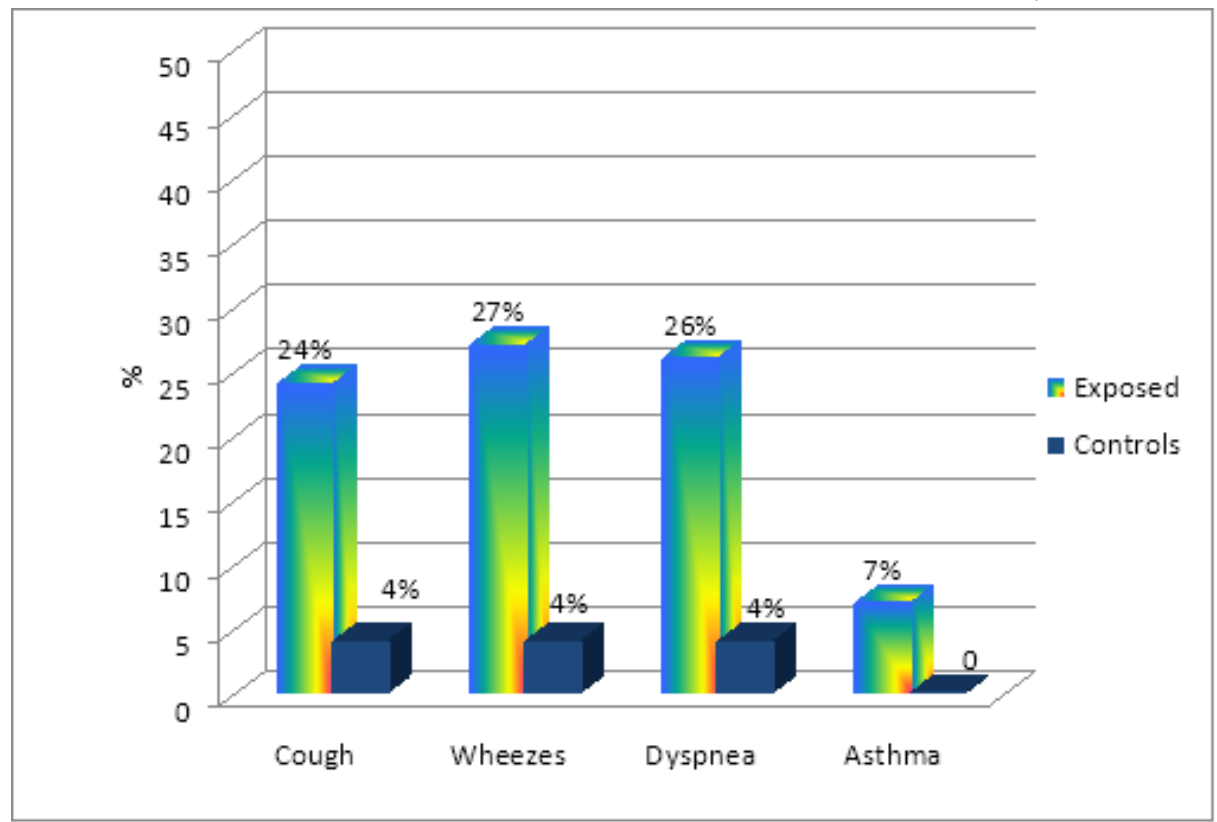

\section{Figure 2}

Prevalence of respiratory manifestations in the exposed at post-season and the control group. 


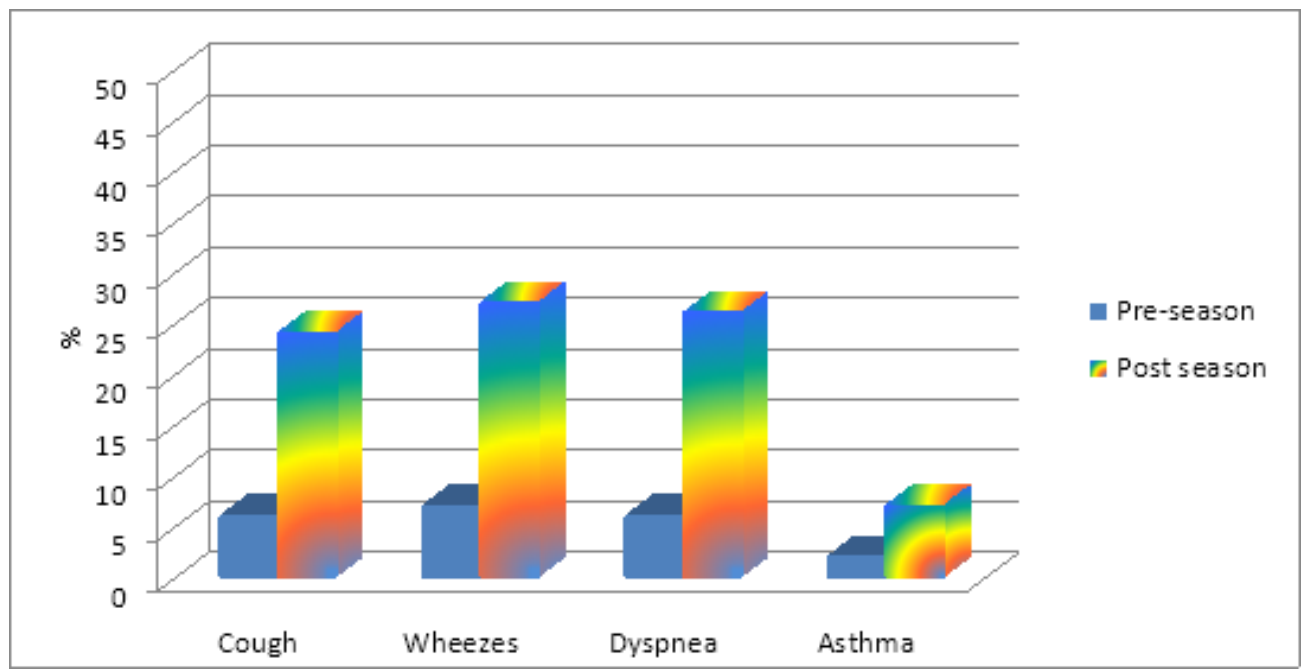

Figure 3

Prevalence of respiratory manifestations at the pre and post season in the exposed group 